\title{
Context-dependent modulation of Pol II CTD phosphatase SSUP-72 regulates alternative polyadenylation in neuronal development
}

\author{
Fei Chen, ${ }^{1,2}$ Yu Zhou, ${ }^{3}$ Yingchuan B. Qi, ${ }^{1,6}$ Vishal Khivansara, ${ }^{4,7}$ Hairi Li, ${ }^{3}$ Sang Young Chun, ${ }^{5}$ \\ John K. Kim, ${ }^{4,8}$ Xiang-Dong Fu, ${ }^{3}$ and Yishi Jin ${ }^{1,2,3}$ \\ ${ }^{1}$ Neurobiology Section, Division of Biological Sciences, University of California at San Diego, La Jolla, California 92093, USA; \\ ${ }^{2}$ Howard Hughes Medical Institute, University of California at San Diego, La Jolla, California 92093, USA $;{ }^{3}$ Department of Cellular \\ and Molecular Medicine, School of Medicine, University of California at San Diego, La Jolla, California 92093, USA; ${ }^{4}$ Life Sciences \\ Institute, Department of Human Genetics, University of Michigan, Ann Arbor, Michigan 48109, USA; ${ }^{5}$ Department of \\ Computational Medicine and Bioinformatics, University of Michigan, Ann Arbor, Michigan 48109, USA
}

\begin{abstract}
Alternative polyadenylation (APA) is widespread in neuronal development and activity-mediated neural plasticity. However, the underlying molecular mechanisms are largely unknown. We used systematic genetic studies and genome-wide surveys of the transcriptional landscape to identify a context-dependent regulatory pathway controlling APA in the Caenorhabditis elegans nervous system. Loss of function in ssup-72, a Ser5 phosphatase for the RNA polymerase II (Pol II) C-terminal domain (CTD), dampens transcription termination at a strong intronic polyadenylation site (PAS) in unc-44/ankyrin yet promotes termination at the weak intronic PAS of the MAP kinase dlk-1. A nuclear protein, SYDN-1, which regulates neuronal development, antagonizes the function of SSUP-72 and several nuclear polyadenylation factors. This regulatory pathway allows the production of a neuron-specific isoform of unc-44 and an inhibitory isoform of dlk-1. Dysregulation of the unc-44 and dlk-1 mRNA isoforms in sydn-1 mutants impairs neuronal development. Deleting the intronic PAS of unc-44 results in increased pre-mRNA processing of neuronal ankyrin and suppresses sydn-1 mutants. These results reveal a mechanism by which regulation of CTD phosphorylation controls coding region APA in the nervous system.
\end{abstract}

[Keywords: pre-mRNA 3' end processing; axon and synapse development; C. elegans; SYDN-1; UNC-44/ankyrin; DLK-1/MAP kinase]

Supplemental material is available for this article.

Received June 1, 2015; revised version accepted October 16, 2015.

Alternative polyadenylation (APA) occurs at polyadenylation [poly(A)] sites (PASs) residing within the $3^{\prime}$ untranslated region (UTR) or in internal introns/exons. mRNAs with long $3^{\prime}$ UTRs generally contain regulatory elements that affect mRNA stability, localization, and translation efficiency. mRNA isoforms with different coding exons produce protein variants that often have distinct or opposing functions (Di Giammartino et al. 2011). Over $50 \%$ of human genes have multiple PASs (Tian et al. 2005). Tissue- and gene-specific APA events are widespread in normal and pathological conditions (Elkon et al. 2013). For

Present addresses: ${ }^{6}$ Institute of Developmental and Regenerative Biology, School of Life and Environmental Sciences, Hangzhou Normal University, 310036 Hangzhou, China; ${ }^{7}$ Children's Research Institute, Department of Pediatrics, University of Texas Southwestern Medical Center, Dallas, TX 75390, USA; ${ }^{8}$ Department of Biology, Johns Hopkins University, Baltimore, MD 21218, USA.

Corresponding author: yijin@ucsd.edu

Article is online at http://www.genesdev.org/cgi/doi/10.1101/gad.266650. 115 . example, rapidly dividing cells or cancer cells preferentially use proximal PASs to produce mRNAs with shorter 3' UTRs than differentiated cells (Sandberg et al. 2008; Mayr and Bartel 2009). In addition, genes with tissue-restricted expression often have single $3^{\prime}$ UTRs, while ubiquitiously transcribed genes generate multiple $3^{\prime}$ UTRs and may use different 3' UTR isoform ratios to achieve tissuespecific expression (Lianoglou et al. 2013).

Nuclear polyadenylation (NpolyA) produces the 3' UTRs of mature mRNAs and involves four major subcomplexes (Colgan and Manley 1997; Millevoi and Vagner 2010; Proudfoot 2011). Cleavage and polyadenylation specificity factors (CPSFs) bind to PASs, which predominantly consist of the AAUAAA hexamer; cleavage stimulation

C 2015 Chen et al. This article is distributed exclusively by Cold Spring Harbor Laboratory Press for the first six months after the full-issue publication date (see http://genesdev.cshlp.org/site/misc/terms.xhtml). After six months, it is available under a Creative Commons License (Attribution-NonCommercial 4.0 International), as described at http:// creativecommons.org/licenses/by-nc/4.0/. 
factors (CstFs) bind to U/GU-rich regions downstream from the cleavage site; and cleavage factors (CFI and CFII) bind to a UGUA motif upstream of the cleavage site. Together with poly(A) polymerase, these complexes coordinate the recognition of cis elements in the $3^{\prime}$ UTRs of pre-mRNA and execute pre-mRNA cleavage and addition of polyadenine. While differential expression of NpolyA core factors or $3^{\prime}$ UTR-binding proteins has been linked to cell type- and tissue-specific APA within 3' UTRs (Licatalosi et al. 2008; Kim et al. 2010b; Hilgers et al. 2012; Jenal et al. 2012; Martin et al. 2012; Masamha et al. 2014), much less is known about how APA at internal intronic/exonic PASs is regulated. During B-cell differentiation, up-regulation of the polyadenylation factor CstF-64 leads to usage of intronic PASs (Takagaki et al. 1996). U1 small nuclear ribonucleoproteins (snRNPs) can prevent premature cleavage and polyadenylation in a splicingindependent manner (Kaida et al. 2010). In neurons, depolarization triggers a transient shortage of U1 snRNPs and prompts the use of internal or proximal PASs (Flavell et al. 2008; Berg et al. 2012).

The C-terminal domain (CTD) of the large subunit of RNA polymerase II (Pol II) is a key regulator of the NpolyA machinery (McCracken et al. 1997; Hirose and Manley 1998). In eukaryotic cells, the CTD consists of 26-52 repeats of the heptapeptide YSPTSPS. The phosphorylation state of each Ser in the heptapeptide has been proposed to function as a "CTD code" that regulates transcription initiation, elongation, and termination/Egloff and Murphy 2008; Buratowski 2009). In particular, Ser2 phosphorylation of the CTD is required for its association with polyadenylation factors in $3^{\prime}$ end formation of pre-mRNA (Hirose and Manley 1998; Licatalosi et al. 2002; Ahn et al. 2004; Meinhart and Cramer 2004; Davidson et al. 2014). Multiple kinases and phosphatases are involved in CTD phosphorylation and dephosphorylation (Meinhart et al. 2005). Among them, the Ssu72 family of phosphatases primarily dephosphorylates Ser5 (Sun and Hampsey 1996; Krishnamurthy et al. 2004). Structural studies show that Ssu72 directly binds the CTD heptapeptide and forms a complex with symplekin (Xiang et al. 2010), a component of the CPSF complex (Kolev et al. 2008; Sullivan et al. 2009). Biochemical studies show that this complex regulates transcription-coupled polyadenylation (Xiang et al. 2010). Yeast Ssu72, the founding member of the family, is essential (Sun and Hampsey 1996). Mammalian Ssu72 is highly expressed in the brain (St-Pierre et al. 2005), but its in vivo function is unknown.

In the nematode Caenorhabditis elegans, most of the conserved components of the NpolyA machinery are essential for embryonic and larval development (Cui et al. 2008). Their roles in the nervous system are largely unknown. The novel nuclear protein SYDN-1 was previously discovered in a genetic enhancer screen for synapse formation and was subsequently shown to negatively regulate $p f_{s-2}$ (Van Epps et al. 2010), the ortholog of human WDR33, an obligatory subunit of the CPSF complex that binds the AAUAAA hexamer (Chan et al. 2014; Schonemann et al. 2014). While the genetic evidence indicates that SYDN-1 inhibits NpolyA activity, it is not known which step of NpolyA and what genes regulated by SYDN-1 are critical for neuronal development.

Here, we identify SSUP-72, the C. elegans ortholog of Ssu72, through analyses of genetic suppressors of sydn-1 mutants. SYDN-1 can bind SSUP-72 and interfere with its association with RNA Pol II. By analyzing the genomic occupancy of Pol II CTD in combination with transgenic reporter assays, we uncovered that SSUP-72 regulates APA at coding region PASs. The interaction of SYDN-1 and SSUP-72 is necessary for the production of the neuron-specific isoform of the ankyrin protein UNC-44 and an inhibitory isoform of the MAPKKK DLK-1. We show that the strength of intronic PASs in unc-44 and $d l k-1$ plays distinct roles for $3^{\prime}$ end processing and transcription termination. Dysregulation of APA of unc-44 or dlk-1 contributes to neuronal defects in sydn-1 mutants. Furthermore, deletion mutations in the coding region PAS of unc-44 cause enhanced pre-mRNA processing of the neuronal isoform and suppress sydn-1 mutants. Taken together, our data reveal a molecular mechanism controlling coding region APA in the nervous system.

\section{Results}

Negative regulation of a subset of NpolyA

factors by SYDN-1 is required for neuronal development in $\mathrm{C}$. elegans

In a genetic screen for regulators of synapse formation, we previously identified the novel nuclear protein SYDN-1 (Van Epps et al. 2010). sydn-1(0)-null mutants exhibit mild behavioral deficits but display strong genetic synergy with other mutants affecting synaptic development. For example, double mutants of sydn-1(0) with syd-2(0)/ a-liprin display severe paralysis, diminished synapse number, and excessive axon branching-phenotypes not found in sydn-1(0) or syd-2(0) single mutants (Van Epps et al. 2010). We took advantage of these synthetic behavioral deficits and isolated a large number of genetic suppressors (Supplemental Material). We characterized multiple mutations that suppressed the synapse and axon morphology defects of $s y d n-1(0)$ but not of syd-2(0) (Fig. 1A-C; Supplemental Fig. S1A). In addition to the previously reported pfs-2/WDR33 (Van Epps et al. 2010), we identified three new genes (Figs. 1C, 2A; Supplemental Table S1): cpsf-4, which encodes the 30-kDa subunit of the CPSF complex; $z f p-3$, which encodes a novel zinc finger protein that can interact with NpolyA proteins; and ssup-72, which encodes the ortholog of CTD Ser5 phosphatase Ssu72. We also tested additional NpolyA genes and found three other genes in which mutations also strongly suppressed sydn-1 (0) (Fig. 1C; Supplemental Fig. S1A,B). These included symk-1/symplekin, a binding partner of Ssu72 and CTD (Xiang et al. 2010), and cids-1 and cids-2, which encode two homologous proteins of the RTT103 family, known to interact with the Ser2 phosphorylated CTD (Kim et al. 2004). In contrast, null or partial loss-of-function alleles of $c p f-1$ and suf-1, encoding CstF50 and CstF77, respectively, and a null mutation of $n r d-1$, which encodes a protein that can bind the Ser5 phosphorylated CTD 
A

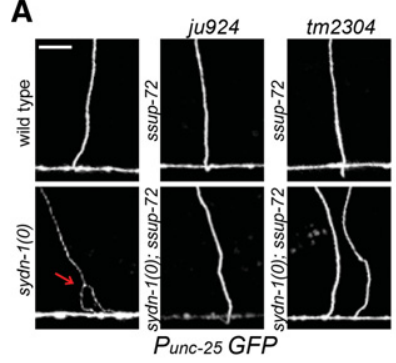

B

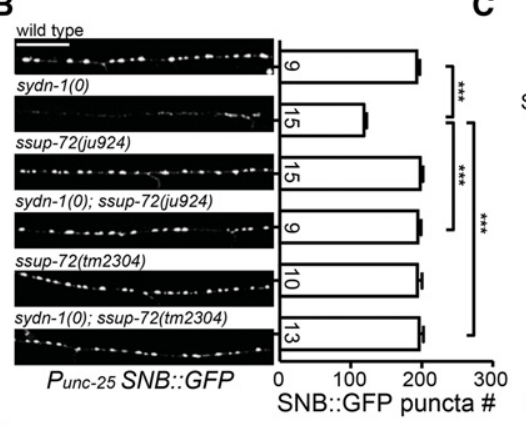

D

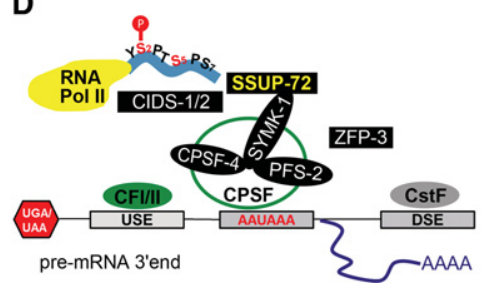

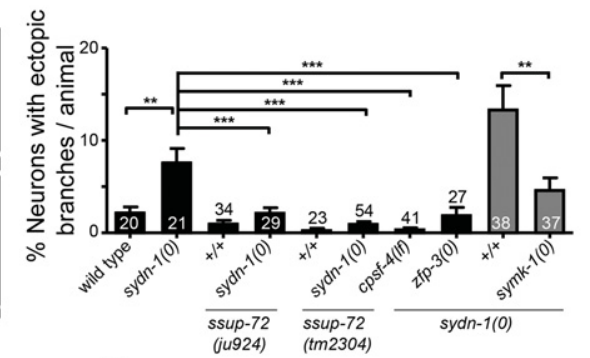

C

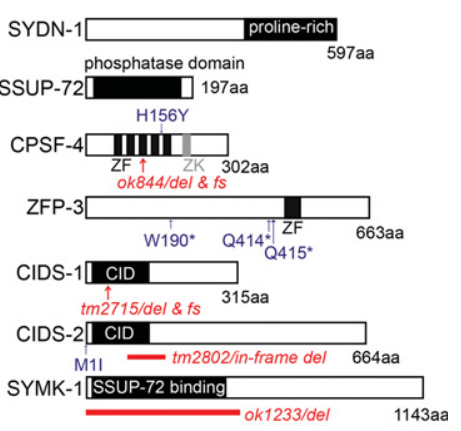

1143aa defects in sydn-1(0). The graph at the right shows the quantification of suppression; data are shown as the total synaptic puncta in the dorsal cord (mean $\pm \mathrm{SEM} ; n=$ number of animals). $\left.{ }^{* * *}\right) P<0.001$, Kruskal-Wallis test and Dunn's multiple comparison post-test. Bar, 20 $\mu \mathrm{m}$. (C) Summary of the proteins and mutations identified from the suppressor analysis of $s y d n-1(0)$. (ZF) Zinc finger domain; (ZK) zinc knuckle domain; (CID) CTD-interacting domain. The SSUP-72-binding domain of SYMK-1 is predicted based on homology (Xiang et al. 2010). (D) Schematic illustration of protein interaction of the $s y d n-1(0)$ suppressor genes based on reported studies on their homologs. One heptapeptide of RNA Pol II CTD is shown, and Ser2 residue is phosphorylated at the 3' end of pre-mRNA. (CFI/II) Cleavage factors; (USE) upstream element; (DSE) downstream element; (AAUAAA) canonical poly(A) signal.

(Vasiljeva et al. 2008), did not suppress sydn-1(0) (Supplemental Fig. S1A,B; data not shown). Thus, the suppression of the neuronal and behavioral defects in sydn-1(0) appears to be highly selective to a subset of the genes in the NpolyA machinery (Fig. 1D).

Single mutants of sydn-1(0) suppressors displayed grossly normal axon and synapse morphology and locomotion and behaved as loss of function. For example, cpsf-4 (ju605) changes a conserved His156 to Tyr in the fourth zinc finger domain of CPSF-4 (Fig. 1C) and suppresses the motor neuron axon branching defects of sydn-1(0); syd-2 (0) animals to the same degree as a null mutation cpsf-4 (ok844) (Supplemental Fig. S1A). Neuron-specific expression of $c p s f-4(+)$ rescued the suppression of cpsf-4(lf) on sydn-1(0) (Supplemental Fig. S1C), supporting a cellautonomous requirement of CPSF-4.

The genetic interaction between sydn-1 and CPSF components raised the possibility that SYDN-1 might affect PAS recognition and/or utilization in general. To address this, we assessed usage of PASs by deep sequencing $3^{\prime}$ ends of mRNAs prepared from stage-matched wildtype and $s y d n-1(0)$ animals (Supplemental Material). We obtained comparable numbers of unique reads of $3^{\prime}$ ends from sydn-1(0) and wild type (Supplemental Fig. S2A) and found that the overall pattern of PAS usage in sydn-
1(0) was similar to wild type (Supplemental Fig. S2B). As transcripts from neuronal genes might generally be low in such samples, we also tested multiple neuronal genes by $3^{\prime} \mathrm{RACE}$ and observed that their PAS usage was not altered in sydn-1(0) (Supplemental Figs. S2C, S3). This analysis suggests that SYDN-1 is unlikely to be required for general recognition of PASs. Instead, SYDN-1 may regulate NpolyA of specific neuronal genes. To dissect this pathway, we focused on ssup-72, as null mutants of ssup-72 completely suppress $s y d n-1(0)$ and are fully viable.

SSUP-72 phosphatase activity is required for its function in SYDN-1-dependent neuronal development

SSUP-72 shares $57 \%$ amino acid identity with human Ssu72 (Fig. 2A). The catalytic nucleophile of human Ssu72 consists of residues Cys12 and Asp143 flanking a two-stranded anti-parallel $\beta$ sheet ( $\beta 2 \mathrm{~A}$ and $\beta 2 \mathrm{~B}$ ) (Xiang et al. 2010). ssup-72(ju924), isolated as a sydn-1(0) suppressor, alters an invariant Gly47 in the loop of the $\beta$ sheet. A null mutation, ssup-72(tm2304), which deletes most of the coding sequence, suppressed the synaptic and axon defects of sydn-1(0) to levels similar to those of ssup-72(ju924) (Fig. 1A,B). Genetic mosaic analysis 
A
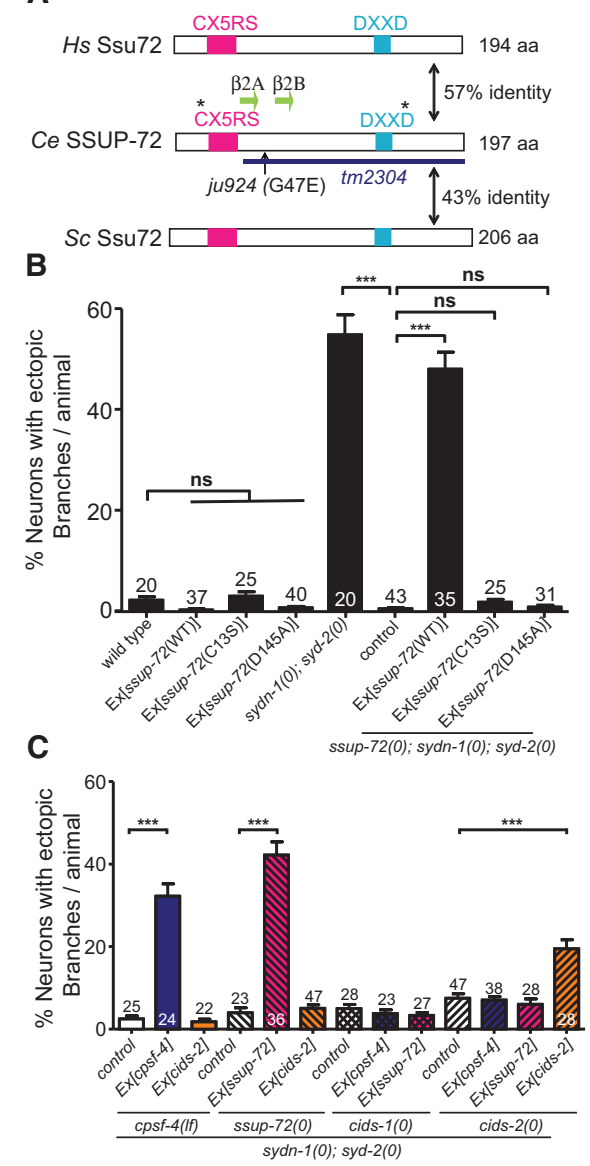

Figure 2. SSUP-72 phosphatase activity is required in the SYDN-1-dependent pathway during neuronal development. $(A)$ Illustration of the SSUP-72 family. The catalytic nucleophile $\left(\mathrm{CX}_{5} \mathrm{RS}\right)$ and aspartate loop (DXXD) are essential for the CTD phosphatase activity. (B) Wild-type but not phosphatase mutant ssup-72 rescues the suppression of sydn-1(0) by ssup-72(0). syd2(0) was used to sensitize detection of the suppression. (C) The function of the suppressor genes of $s y d n-1(0)$ is dependent on each other. Transgenes overexpressing ssup-72, cpsf-4, or cids-2 cannot bypass the requirement of other suppressor genes. Analysis of axonal branches in GABAergic motor neurons in $B$ and $C$ was the same as in Figure 1A. $\left(^{* * *}\right) P<0.001$, Kruskal-Wallis test and Dunn's multiple comparison post-test.

and neuron-specific expression studies indicate that SSUP-72 functions cell-autonomously (Supplemental Fig. S4A,B), similar to CPSF-4 (Supplemental Fig. S1C) and PFS-2 (Van Epps et al. 2010).

The phosphatase activity of human Ssu 72 is required for transcription-coupled polyadenylation in vitro when in a complex with symplekin (Xiang et al. 2010), whereas, in yeast, Ssu72 can affect $3^{\prime}$ end processing independently of its phosphatase activity (Krishnamurthy et al. 2004). To test whether the phosphatase activity of SSUP-72 is required for its function, we mutated the catalytic Cys13 or Asp145 to Ser or Ala, respectively (Fig. 2A). Expression of either mutant SSUP-72 (C13S or D145A) failed to rescue ssup-72(0); sydn-1(0); syd-2(0) and did not cause discern- able defects in a wild-type background (Fig. 2B). Moreover, Western blot analysis using the $4 \mathrm{H} 8$ antibody, which was raised against Ser5P-CTD, showed increased CTD phosphorylation in ssup-72(0) and sydn-1(0); ssup-72(0) animals (Supplemental Fig. S4C-E).

The cyclin-dependent kinase Cdk7 can phosphorylate Ser5 of Pol II CTD (Phatnani and Greenleaf 2006). To further address whether regulated CTD Ser5 phosphorylation is involved in neuronal development, we examined $c d k-7(a x 224 t s)$, a partial loss-of-function mutation that displays decreased Ser2 and Ser5 phosphorylated CTD and maternal effect lethality at the restrictive temperature (Wallenfang and Seydoux 2002). cdk-7(ax224ts) mutants raised at the restrictive temperature showed grossly normal neuronal morphology (Supplemental Fig. S4G; data not shown) and strongly enhanced the axonal and behavioral defects of sydn-1(0) (Supplemental Fig. S4G), suggesting that reducing phosphorylated CTD impairs neuronal development in a manner sensitive to the function of $s y d n-1$. Double mutants of $c d k-7(a \times 224 t s)$; ssup-72(0) showed no obvious neuronal defects (Supplemental Fig. S4G). Notably, the maternal effect lethality of $c d k-7$ (ax224ts) at the restrictive temperature was partially suppressed by ssup-72(0) (Supplemental Fig. S4F). Taken together, these data provide further support for the conclusion that SSUP-72 phosphatase acts on the Ser5 phosphorylated CTD.

Since orthologs of the genes identified as $s y d n-1(0)$ suppressors are known to interact biochemically (Dichtl et al. 2002), we next addressed their functional dependency. Overexpression of ssup-72(+) or cpsf-4(+) rescued the suppression of $s y d n-1(0)$ by ssup-72(0) or cpsf-4(lf), respectively, but did not rescue the suppression by cids-1(0) or cids-2(0) (Fig. 2C). Conversely, overexpression of cids-2(+) rescued the suppression of $s y d n-1(0)$ by cids2(0) but not that by ssup-72(0) or cpsf-4(lf) (Fig. 2C). These data suggest that the proteins encoded by these sydn-1(0) suppressor genes act in a mutually dependent manner.

\section{SYDN-1 can bind SSUP-72 and be coimmunoprecipitated with RNA Pol II}

The genetic interaction between sydn-1 and multiple NpolyA factors suggests that SYDN-1 inhibits the function of NpolyA factors. We next addressed how SYDN-1 might regulate SSUP-72. The overall levels of ssup-72 mRNA were similar in sydn-1(0) and wild type (Supplemental Fig. S5B). Animals expressing a GFP::SSUP-72 translational fusion protein showed nuclear localization in multiple tissues, including the epidermis, intestine, and nervous system (Supplemental Fig. S5A); this pattern was not altered in sydn-1(0) mutants (Supplemental Fig. S5C). Similarly, sydn-1(0) did not alter the expression pattern of CPSF-4 (Supplemental Fig. S5D).

We next tested whether SYDN-1 might physically interact with SSUP-72. In the yeast two-hybrid assay, SYDN-1 interacted with SSUP-72, and this interaction involved the C-terminal half of SYDN-1 (amino acids 301-578) (Fig. 3A). As Ssu72 is known to bind the CTD of RNA Pol II 
A

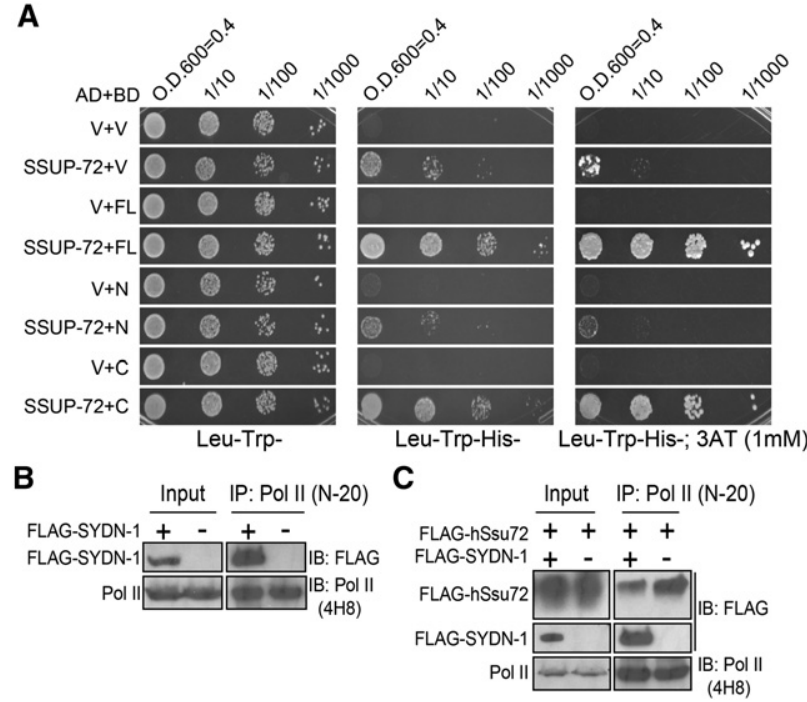

Figure 3. SYDN-1 can bind SSUP-72 and associate with RNA Pol II. (A) Interaction between SYDN-1 and SSUP-72 in a yeast two-hybrid assay. The SSUP-72 full-length protein fused to the GAL4 activation domain (AD) interacts with the SYDN-1 fulllength protein or the CTD fused to the GAL4-binding domain (BD) and does not interact with the SYDN-1 N-terminal domain or GAL4-binding domain. Yeast cells were plated in a dilution series on selection plates of $\operatorname{Trp}^{-} \mathrm{Leu}^{-}(1 e f t), \mathrm{Trp}^{-} \mathrm{Leu}^{-} \mathrm{His}^{-}$(middle), and $\operatorname{Trp}^{-} \mathrm{Leu}^{-} \mathrm{His}^{-}$with $1 \mathrm{mM} 3 \mathrm{AT}$. (V) Empty vector; (FL) SYDN-1 full-length protein; (N) SYDN-1 (1-300 amino acids); (C) SYDN-1 (301-578 amino acids). (B) SYDN-1 can associate with human RNA Pol II. HEK293T cells were transfected with Flag-tagged SYDN-1, and cell lysates were immunoprecipitated with N-20 anti-Pol II antibodies recognizing the large subunit of RNA Pol II. Coimmunoprecipitated proteins were blotted with anti-Flag and 4H8 anti-Pol II CTD. Two percent of cell lysates were run as the input. $(C)$ The presence of SYDN-1 can reduce the association of human Ssu72 with Pol II. HEK293T cells were transfected with the constructs indicated, and cell lysates were immunoprecipitated with $\mathrm{N}-20$ anti-Pol II antibodies. Coimmunoprecipitated proteins were blotted with anti-Flag and 4H8 anti-Pol II CTD. One percent of immunoprecipitation lysates were run as the input.

(Xiang et al. 2010), we asked whether SYDN-1 could also associate with RNA Pol II. Because SYDN-1 expressed in C. elegans was highly insoluble (data not shown), we expressed Flag::SYDN-1 in HEK293T cells and found that it localized to subnuclear puncta (Supplemental Fig. S5E), consistent with its nuclear localization in C. elegans neurons (Van Epps et al. 2010). We then immunoprecipitated RNA Pol II using the N-20 antibody, which recognizes the large subunit of human RNA Pol II (Cheng and Sharp 2003), and consistently pulled down Flag::SYDN-1 (Fig. 3B). Human Ssu72 could also be coimmunoprecipitated with RNA Pol II (Fig. 3C). Interestingly, when we coexpressed SYDN-1 with human Ssu72, significantly less Ssu72 was coimmunoprecipitated with RNA Pol II (Fig. 3C). While it is possible that heterologous expression of SYDN-1 might lead to a dominant-negative effect of titrating human Ssu72, together with the genetic analysis and the observed protein interaction in yeast two-hybrid assay, these data suggest that SYDN-1 may interfere with the interaction between RNA Pol II and Ssu72.

Genome-wide analysis of RNA Pol II CTD occupancy identifies neuronal ankyrin UNC-44 as a target of SYDN-1 and SSUP-72

As the phosphorylation state of the RNA Pol II CTD is intimately linked to transcription (Buratowski 2009), we next examined CTD occupancy by performing chromatin immunoprecipitation (ChIP) using the 4H8 antibody followed by deep sequencing (ChIP-seq) (Supplemental Fig. S6A; Supplemental Material). We obtained comparable numbers of reads ( 20 million for each) from ChIP-seq DNA libraries made from stagematched worms of wild type; sydn-1(0) and ssup-72(0) single mutants; and sydn-1(0); ssup-72(0) double mutants (Supplemental Table S5). We mapped the unique reads to the C. elegans genome. About 4000 proteincoding genes exhibited Pol II occupancy with largely overlapping gene coverage among the four strains (Supplemental Figs. S6B, S7A,C).

The intergenic regions for many genes in C. elegans are generally short, with the transcription end site (TES) or transcriptional start site (TSS) of a gene often overlapping with neighboring genes. Through both manual inspection of the entire genome and bioinformatic analyses of wellannotated genes, we compared the CTD occupancy at nonoverlapping TESs (Supplemental Material). For most genes, the CTD occupancy pattern within \pm 500 base pairs (bp) flanking each TES was similar between wild type and sydn-1(0) (Supplemental Figs. S6C, S7B; Supplemental Excel Files S1-S3). Among the few genes with significant changes in CTD occupancy between sydn-1(0) and wild type, the ankyrin gene unc-44 attracted our interest because it is known to undergo APA in neurons.

The unc-44 locus spans $>40 \mathrm{~kb}$ and generates multiple mRNA isoforms due to alternative TSSs, splicing, and polyadenylation (Fig. 4A; Boontrakulpoontawee and Otsuka 2002; Otsuka et al. 2002). unc-44 mRNA isoforms encode several proteins containing the ankyrin domain in the $\mathrm{N}$ terminus. The last three exons encode a large protein domain unique to the neuronal ankyrin isoform UNC44F. By careful inspection of Pol II CTD occupancy, we noticed a marked reduction of Pol II CTD binding at the last three exons of unc-44 in sydn-1(0) compared with wild type, whereas Pol II CTD occupancy in the $5^{\prime}$ region of the gene was unchanged in sydn-1(0) (Fig. 4A; Supplemental Fig. S6C-E). ssup-72(0) showed normal Pol II CTD occupancy and suppressed the reduced Pol II CTD binding in sydn-1(0) (Supplemental Figs. S6C,D, S7B-D). We quantitated mRNA levels of unc-44 using RT-qPCR. In sydn-1 (0), levels of unc-44f mRNA encoding the neuronal isoform were decreased to $<10 \%$ of wild type, and this reduction was restored to above the wild-type levels in the ssup-72(0); sydn-1(0) double mutants (Fig. 4B). The levels of unc-44 mRNA amplified using primers targeting a common region (unc-44c) were not significantly different among wild-type, sydn-1(0), ssup-72(0), and sydn-1(0); 


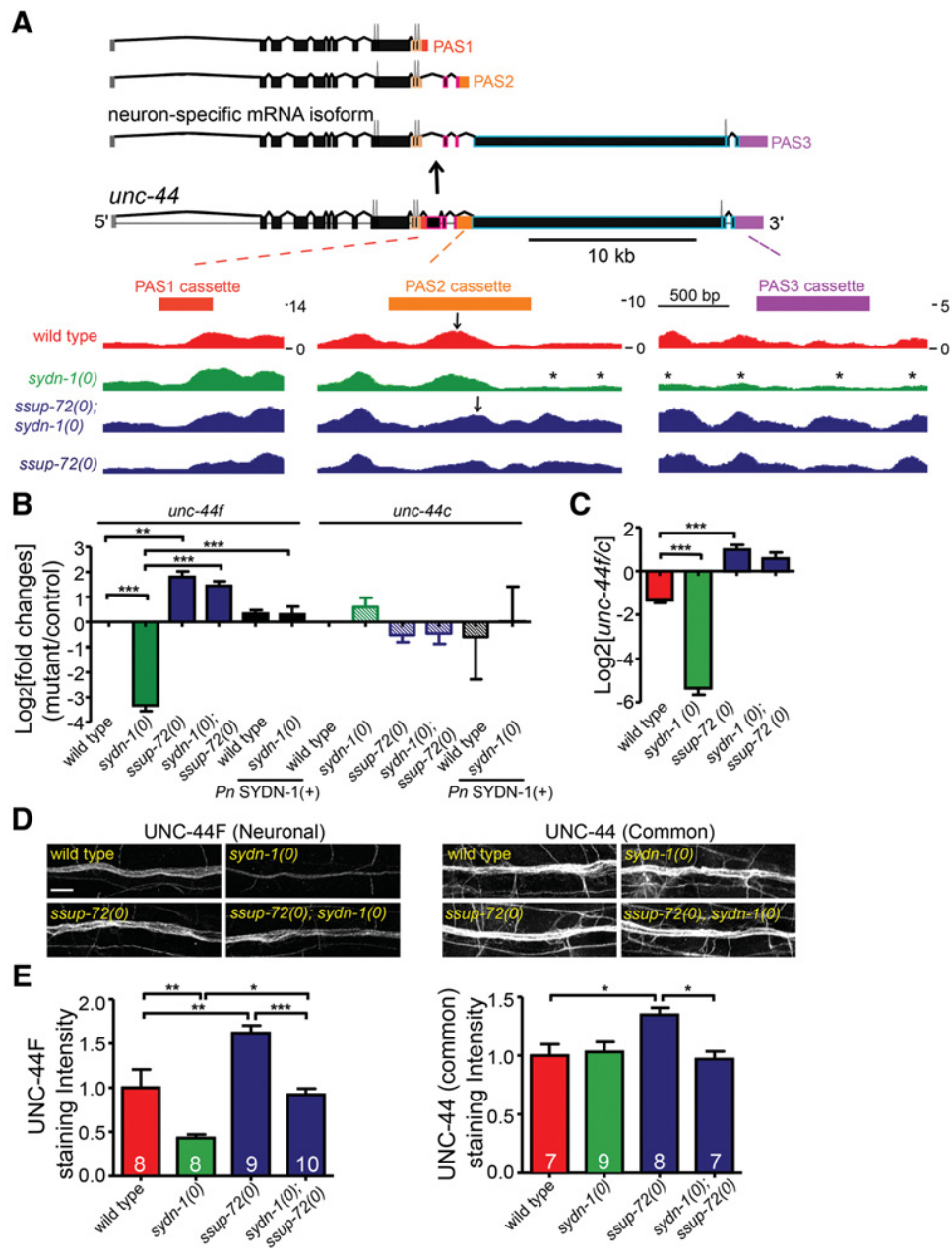

Figure 4. The expression of the neuron-specific unc-44f long isoform depends on SYDN-1 and SSUP-72. (A) 4H8 ChIP-seq analyses show a SYDN-1- and SSUP-72-dependent RNA Pol II CTD-binding pattern at the unc-44 locus. sydn-1(0) shows a greater than twofold significant decrease in Pol II CTD binding in the coding region of the neuron-specific unc-44f isoform compared with wild type (Supplemental Material), which is reversed by ssup-72(0). (Top) unc-44 locus produces transcripts terminating at three PASs, and the usage of PAS3 is neuron-specific. Three PAS regions are enlarged below, and a few exons preceding the PAS cassette are outlined in colors. Black arrows indicate Pol II pausing at the unc44 PAS2 cassette. Asterisks indicate reduced Pol II binding after unc-44 PAS2 in sydn-1(0). (B) SYDN-1 and SSUP-72 interaction regulates the mRNA levels of unc$44 f$. cDNA libraries were constructed from mRNAs isolated from synchronized L2 worms. RT-qPCR analyses show that unc-44f in sydn-1(0) (solid bars) is reduced to $<10 \%$ that of wild type; loss of function in ssup-72 and pan-neuronal expression of sydn-1 completely reversed this reduction. Total levels of unc-44 common isoforms (slashed bars; unc-44c) show mild change. The histogram shows quantification of both unc-44f and unc-44c [for wild type and sydn-1(0), $n=9$; for ssup-72(0) and ssup72(0); sydn-1(0), $n=6$; for Pn-sydn-1 and sydn-1(0); Pnsydn-1, $n=3$ ]. rps-25 was used as the internal control. Shown are the $\log _{2}$ values of fold changes between mutant strains and wild type. $\left(^{* *}\right) P<0.01$; $\left(^{* * *}\right) P<0.001$, one-way ANOVA and Bonferroni's multiple comparison post-test. $(C)$ ssup-72(0) further increases the mRNA levels of unc-44f. The ratio between unc- $44 f$ and unc- $44 c$ is decreased in sydn-1(0) and increased in ssup-72(0) and ssup-72(0); sydn-1(0) compared with wild type. Shown is the $\log _{2}$ value of the ratios between unc-44f and unc$44 c .\left(^{* * *}\right) P<0.001$, one-way ANOVA and Bonferroni's multiple comparison post-test. $(D)$ Protein levels of the neuron-specific UNC-44F isoform is reduced in sydn-1 (0). Shown are confocal images of immunostaining of UNC-44 protein in the ventral cord in the genotypes indicated. Bar, $10 \mu \mathrm{m}$. (E) Quantification of UNC-44 immunostaining in the genotypes indicated. Confocal $Z$-stack images of the nerve cords $(0.5-\mu \mathrm{m}$ section, 15 sections per cord) were collected for quantitative analysis. Fluorescence intensities in different genotypes were normalized to the average intensity of wild type. Shown are normalized intensities \pm SEM $n=$ number of animals. $\left.\left({ }^{*}\right) P<0.05 ;\left({ }^{* *}\right) P<0.01 ;{ }^{* * *}\right) P<0.001$, one-way ANOVA and Bonferroni's multiple comparison post-test.

ssup-72(0) animals (Fig. 4B). Moreover, pan-neuronal expression of sydn-1(+) fully rescued the reduction of unc$44 f$ mRNA in sydn-1(0) (Fig. 4B). Thus, the production of the neuron-specific unc-44f mRNA isoform is dependent on neuronal expression of sydn-1.

To examine how the sydn-1 pathway affected UNC-44 protein levels, we performed immunostaining using antibodies raised against the neuronal UNC-44F-specific domain or against the common region of all UNC-44 isoforms, which are expressed in many tissues (Otsuka et al. 2002). We quantitated UNC-44 staining intensity in the ventral nerve cord and found that UNC-44F-specific staining was decreased dramatically in $s y d n-1(0)$ compared with wild type and that this decrease was restored to wild-type levels in the sydn-1(0); ssup-72(0) double mutants (Fig. 4D,E, left panel images and left histogram). In agreement with the RT-qPCR results, the immunostaining intensity for common UNC-44 isoforms was unaffected in sydn-1(0) single mutants (Fig. 4D,E, right panel images and right histogram). Together, these analyses show that SYDN-1 is specifically required for expression of the neuronal ankyrin UNC-44F isoform via its interaction with SSUP-72.

\section{Loss of ssup-72 function prevents transcription termination at a strong PAS in the coding region of unc-44}

By 3'RACE analyses, we validated two major PASs within the coding region of unc-44, for simplicity named PAS1, PAS2 (present in mRNAs expressed in many tissues), and PAS3 (present only in the neuronal unc- $44 f$ mRNAs) (Otsuka et al. 2002; Supplemental Fig. S8A,B). All three PASs are composed of the canonical PAS AAUAAA (Supplemental Table S4). We analyzed 3'RACE products and found that $s y d n-1(0)$ or ssup-72(0) alone did not alter the use of poly(A) addition sites of unc-44 transcripts (Supplemental Fig. S8A,B). In sydn-1(0) animals, the amount 
of PAS3 usage was noticeably reduced, while those of PAS1 and PAS2 showed no detectable changes from wild type (Supplemental Fig S8A-C). Interestingly, by RT-qPCR analyses, we found that, in ssup-72(0) and sydn-1(0); ssup-72(0), the ratio between the unc-44f and unc-44c isoforms was higher than that in wild type (Fig. $4 \mathrm{C}$, right panel). Moreover, Pol II CTD pausing at the endogenous PAS2 region of unc-44 was noticeably shifted in ssup-72(0) animals (Figs. 4A, 5A; Supplemental Fig. S9A, $\mathrm{B})$, suggesting that production of unc- $44 f$ may be dependent on the regulation of internal PASs.

To specifically examine how sydn-1 and ssup-72 regulate $3^{\prime}$ end processing of unc-44 mRNA in neurons, we next generated a set of mini-expression constructs driven by a pan-neuronal promoter (Supplemental Table S2). In these constructs, the sequences of unc-44 PAS1 or PAS2 cassettes, including the corresponding terminal introns, were placed downstream from the GFP coding sequence (Fig. 5B,C). To mimic the competition between internal and distal PASs, we placed the $3^{\prime}$ end sequences of the muscle myosin unc-54 gene downstream from the unc-44 internal PAS1 or PAS2 cassettes, respectively (Fig. 5B,C). CTD occupancy at the endogenous unc-54 PAS locus was not dependent on SYDN-1 or SSUP-72 (data not shown). To precisely compare transcription-coupled $3^{\prime}$ end processing from these reporters in vivo, we generated single-copy integrated transgenes (Mos-SCI) at the same locus (Supplemental Table S2).
We verified by $3^{\prime} \mathrm{RACE}$ analysis that, in wild-type animals, the usage of PAS1 and PAS2 in the minireporters recapitulated that at the endogenous unc-44 locus (Fig. 5B, C). We did not detect usage of the downstream unc-54 3' PAS in either reporter, indicating that these two internal PASs are strong signals for $3^{\prime}$ end processing and transcription termination. sydn-1(0) did not alter the usage of either PAS1 or PAS2 in these reporters (Fig. 5B,C). However, in ssup-72(0) or ssup-72(0); sydn-1(0) mutants, we observed increased usage of the downstream unc-54 PAS from the PAS2 reporter (Fig. 5C). The PAS1 reporter showed no changes in ssup-72(0) or ssup-72; sydn-1(0) (Fig. 5B). These data suggest that loss of ssup-72 function caused compromised transcription termination at PAS2. Based on the observation that transcription from the PAS2 reporter proceeds to the distal unc-54 PAS when ssup-72 is absent, we infer that inhibition of SSUP-72 phosphatase activity by SYDN-1 at the PAS2 region of the endogenous unc-44 locus is required for producing the neuron-specific unc- $44 f$ isoform.

\section{Dysregulation of unc-44f transcripts contributes to the} neuronal phenotypes of sydn-1 mutants

To examine whether dysregulated APA of unc-44 might contribute to the neuronal defects in sydn-1(0), we next performed genetic interaction studies. The long isoform of unc-44 is required for axonogenesis

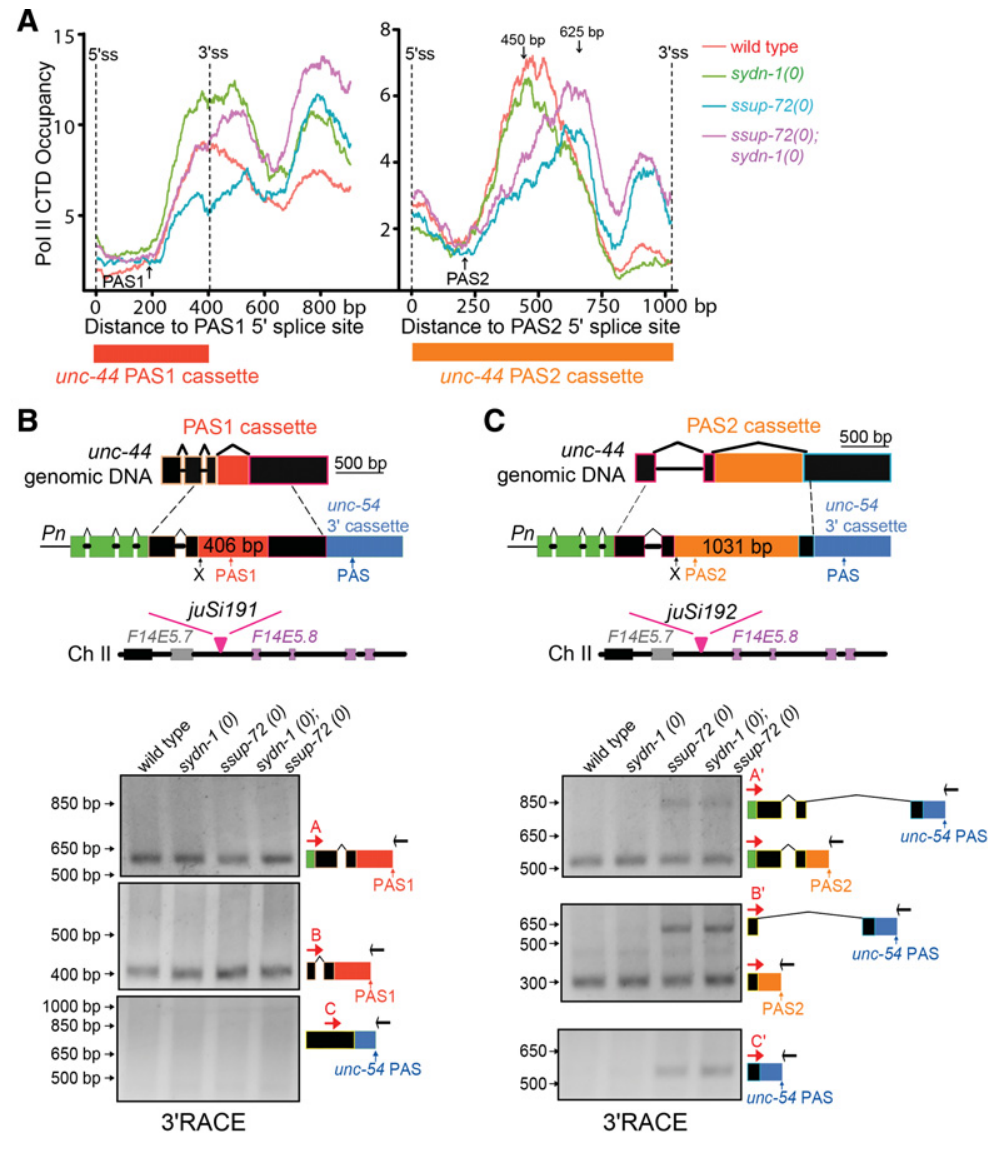

Figure 5. SSUP-72 regulates $3^{\prime}$ end processing at the strong internal PAS2 of unc-44. (A) Shown are RNA Pol II CTD occupancy around the PAS1 (red bar below the left graph) and PAS2 (orange bar below the right graph) cassettes of unc-44 in wild type and mutants as indicated. 5'ss and 3'ss and the dashed lines denote the $5^{\prime}$ and $3^{\prime}$ splice sites flanking each cassette. Peak location of RNA Pol II CTD occupancy in the PAS2 cassette is around the 450-bp position ( $5^{\prime}$ splice site being $0)$ in wild type and $s y d n-1(0)$ and is shifted to $625 \mathrm{bp}$ in ssup-72(0) and ssup-72(0); sydn-1(0). (B) sydn-1 and ssup-72 do not affect the usage of the internal PAS1 of unc-44. (Top panel) Schematic illustration of the GFP reporter for the unc-44 PAS1 cassette ( juSi191) inserted on chromosome II. (Bottom panel) Images of DNA electrophoresis gels of $3^{\prime} \mathrm{RACE}$ results of the PAS1 reporter using three different gene-specific primers (red arrows) and reverse adaptor primer (black arrows) in the genotypes indicated; the illustrations represent the sequences of the PCR fragments. No products were amplified for primer C. $(C)$ Loss of function in ssup-72 results in pre-mRNA $3^{\prime}$ end processing at the distal unc-54 PAS in addition to the internal PAS2 of unc-44. (Top panel) Schematic illustration of the GFP reporter for the unc-44 PAS2 cassette (juSi192) inserted on chromosome II. (Bottom panel) Images of DNA electrophoresis gels of 3'RACE results of the PAS2 reporter using three different gene-specific primers (red arrows) and reverse adaptor primer (black arrows) in the genotypes indicated; illustrations represent the sequences of the PCR fragments. (X) Stop codon. 
(Boontrakulpoontawee and Otsuka 2002). unc-44 (tm349) is a deletion within the large unc-44f-specific exon, and homozygous mutants are severely uncoordinated and slow-growing. The axonal defects in unc-44 (tm349) animals resembled those in unc-44(e362), a mutation affecting all isoforms (Boontrakulpoontawee and Otsuka 2002). To test whether reducing neuronal UNC44F contributes to the $s y d n-1(0)$ phenotype, we constructed sydn-1(0); unc-44(tm349)/nT1 (nT1 is a chromosomal balancer for propagation). By RT-qPCR analysis, we observed significant reduction of unc-44f in the unc-44 (tm349)/nT1 animals compared with controls (Fig. 6A). Based on the RT-qPCR analysis (Fig. 4B), we estimated that, in sydn-1(0), the unc-44f mRNA levels were $<10 \%$ of wild-type levels. We predicted that any further decrease of unc-44f in the unc-44(tm349)/nT1 heterozygotes should induce a more severe phenotype (Fig. 6A). Indeed, heterozygosity for unc-44(tm349) significantly enhanced the axon branching phenotype in $s y d n-1(0)$ but had no effect in a sydn-1(+) background (Fig. 6B). As a control for the specificity of unc- $44 f$ dosage-dependent enhancement of axon defects on $s y d n-1(0)$, we tested syd-2(0) mutants and observed no discernable difference in axon morphology among animals containing $n T 1 /+$ or unc-44(tm349)/ $n T 1$ (Fig. 6B). The sensitivity of sydn-1(0) to reduced unc-44 gene dosage is consistent with the sydn-1(0) mutant displaying lower UNC-44F expression.

We also addressed whether removing PAS2 of unc-44 could increase the expression of unc- $44 f$ mRNA, thereby suppressing sydn-1(0). Using CRISPR genome-editing technology, we generated two mutations, ju1377 and ju1378, which removed 83 bp and 180 bp containing the polyadenylation consensus sequence in the PAS2 cassette, respectively (Fig. 6C; see the Materials and Methods). Both alleles showed increased production of unc-44f by $3^{\prime}$ RACE (Fig. 6C) and suppressed the movement defects of sydn-1 (0); syd-2(0) double mutants (Materials and Methods). Single mutants of either allele showed normal neuronal morphology and strongly suppressed the axon morphology defects of sydn-1(0) (Fig. 6D). Thus, we conclude that pre-mRNA processing of unc-44f in neuronal development depends critically on the regulation of internal PAS2 by the SYDN-1 and SSUP-72 pathway.

\section{Usage of a weak coding region PAS of dlk-1 also depends on SYDN-1 and SSUP-72}

To explore whether the SYDN-1 pathway might have a broader role in regulating APA, we tested several other neuronal genes that produce multiple mRNA isoforms by APA

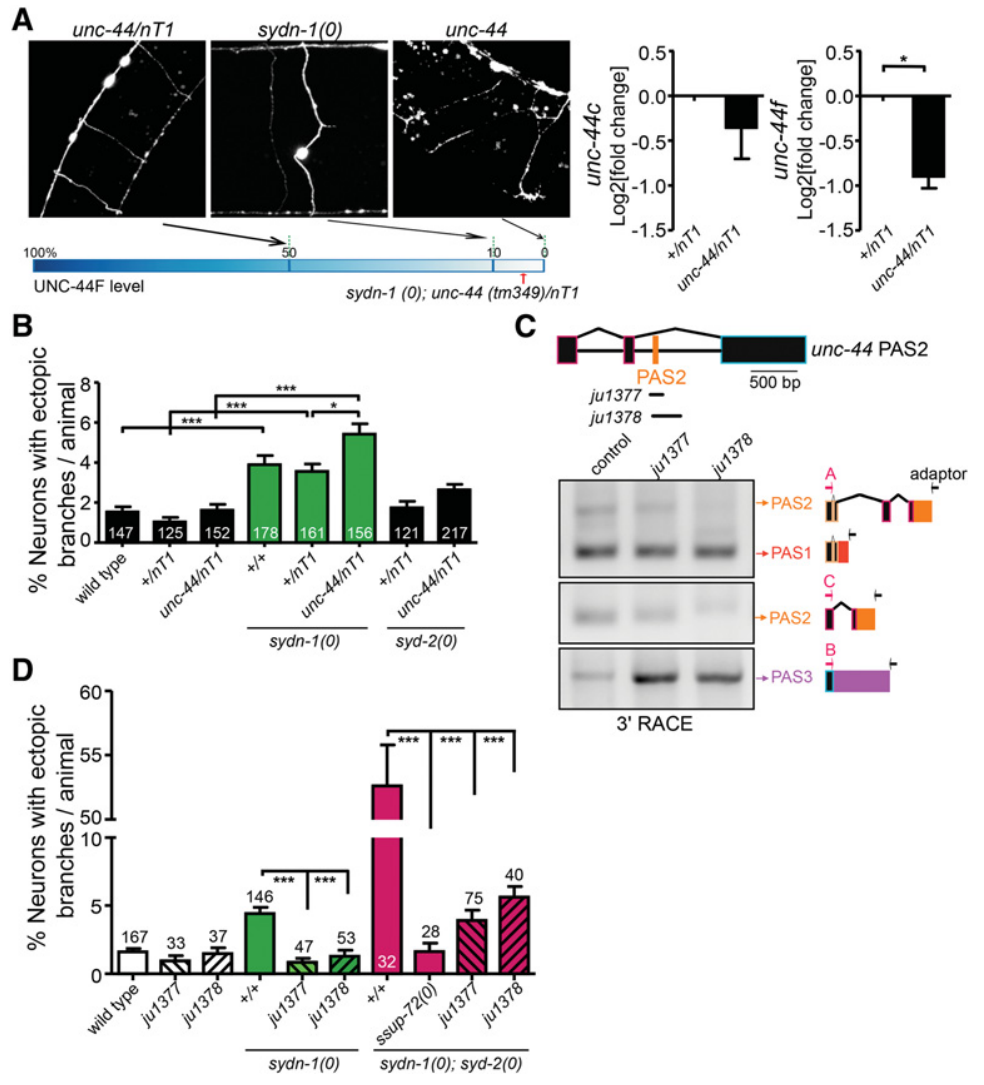

Figure 6. Dysregulated APA of unc-44 causes neuronal defects of sydn-1 mutants. (A) Levels of unc- $44 f$ mRNA correlate with axonal morphology defects. Shown at the left are images of axonal morphology of the GABAergic motor neurons labeled by juIs 76 [Punc-25-GFP] in the genotypes indicated. Below is a diagram depicting the functional correlation to the levels of UNC-44F in sydn-1 and unc-44. The two graphs at the right show the mRNA levels of unc-44c and neuron-specific unc$44 f(n=3)$. Shown is the $\log _{2}$ value of fold changes between $+/ n T 1$ and $t m 349 / n T 1$ strains. $\left({ }^{*}\right) P<0.05$, paired $t$-test. rps-25 was used as the internal control. $(B) \mathrm{Re}$ duced unc-44f dosage in sydn-1(0) mutants induces more severe defects in neurons. The graph shows quantification of the average percentage of abnormal neurons with ectopic axonal branches per animal. Data are shown as mean \pm SEM; $n=$ number of animals. (*) $P<0.05$; $\left(^{* * *}\right) P<0.001$, Kruskal-Wallis test and Dunn's multiple comparison post-test. $(C)$ Deletion mutations of the PAS2 at the endogenous unc-44 locus cause increased polyadenylation at PAS3 of unc-44 compared with sydn-1(0). The top illustration shows the location of ju1377 and ju1378 mutations around PAS2 (also see Supplemental Table S4). Images show 3'RACE analyses of unc-44 transcripts in sydn-1(0); syd-2(0) (left lane), unc-44(ju1377); sydn-1(0); syd-2 (middle lane), and unc-44(ju1378); sydn-1(0); syd-2(0) (right lane). Black arrows are the reverse adaptor primer commonly used in the 3'RACE kit; gene-specific primer A amplifies both PAS1 and PAS2, gene-specific primer B amplifies PAS3, and gene-specific primer $\mathrm{C}$ amplifies PAS2. Sequences of the amplified DNA fragments corresponding to each band are shown as an illustration. $(D)$ Ectopic branches of GABAergic motor neurons in sydn-1(0) are suppressed by deletion mutations of unc-44 PAS2. Shown is the quantification of the average percentage of GABAergic neurons that display ectopic branches in the genotypes indicated. Fifteen to 19 commissures per adult animal were counted. $\left(^{* * *}\right) P<0.001$, Kruskal-Wallis test and Dunn's multiple comparison post-test. Bar, $10 \mu \mathrm{m}$. 
and identified a distinct mode of regulation in the dlk-1 gene, which encodes a conserved MAPKKK (Nakata et al. 2005). dlk-1 produces two protein isoforms, DLK-1L (long) and DLK-1S (short) (Yan and Jin 2012), with dlk-1S transcripts generated using an intronic PAS (Fig. 7A). While the PAS usage of $d l k-1 L$ showed no difference between wild type and sydn-1(0), dlk-1S PAS usage was virtually undetectable in sydn-1(0) (Supplemental Fig. S3). Moreover, transgenic reporters containing GFP::DLK-1S coding sequences followed by the $d l k-1 S 3^{\prime}$ end cassette showed consistently decreased GFP levels in sydn-1(0) compared with wild type, and ssup-72(0) suppressed this reduction in GFP expression (Supplemental Fig. S10A, left panel and histogram, juEx5216). Neither sydn-1(0) nor ssup-72(0) affected expression of a control transgene with the unc-54 PAS cassette (Supplemental Fig. S10A, right panel and histogram, $j u E x 5272)$. These results suggest that SYDN-1 promotes the expression of $d 1 k-1 S$.

To further test how the usage of the $d l k-1 S$ PAS was affected in sydn-1(0) and ssup-72(0), we generated an integrated single-copy $d l k$ - $1 S$ PAS reporter transgene containing the $d l k-1 S 3^{\prime}$ end cassette flanked by exon 7 and exon 8 sequences and followed by the unc-54 PAS cassette (Supplemental Fig. S10B, juSi186, left panel). 3'RACE analysis of this $d l k-1 S$ PAS reporter identified one major product that corresponded to the spliced transcripts ending with the unc-54 PAS (Supplemental Fig. S10B, right panel). This observation indicates that the $d l k-1 S$ PAS is a weak signal for $3^{\prime}$ end processing and may be outcompeted by the splicing of $d l k-1$ intron 7.

To sensitize the detection of the $d 1 k$-1S PAS, we mutated the splice sites of intron 7 (Fig. 7A, juSi243, left panel). By 3'RACE analyses, we obtained multiple products generated from the juSi243 line, and analyses of their se- quences showed that these transcripts terminated at either the $d l k-1 S$ PAS or the PAS of a neighboring gene, F14E5.8, located $3^{\prime}$ to the integration site (Fig. 7A). In wild type, $19 \%$ of the DNA clones $(n=22)$ showed usage of the dlk-1S PAS, comparable with sydn-1(0) (14\% colonies, $n=22$ ) (Fig. 6C, right panel). Interestingly, ssup-72(0) greatly increased the usage of the dlk-1S PAS in juSi243, as, in ssup-72(0) single and ssup-72(0); sydn-1(0) double mutants, $d l k-1 S$ PAS usage was increased to $40 \%$ ( $n=$ $23)$ and $60 \%(n=20)$, respectively (Fig. 7A, right panel). These data are consistent with the model that SYDN-1 promotes the usage of the weak $d l k-1 S$ PAS by inhibiting SSUP-72 function.

DLK-1S binds and inhibits DLK-1L (Yan and Jin 2012). Decreasing levels of DLK-1S elevate the activity of DLK1L. To address whether up-regulation of DLK-1L activity contributed to the sydn-1(0) phenotype, we generated double mutants of sydn-1(0); dlk-1(0). dlk-1(0) suppressed the ectopic axonal branches of sydn-1(0) single and sydn-1 (0); syd-2(0) double mutants (Fig. 7B). These data support a conclusion that the aberrant axon morphology of the sydn-1(0) mutant likely results from dysregulation of APA in multiple neuronal genes and underscore the complexity of context-dependent APA.

\section{Discussion}

APA empowers regulation of gene expression and cellular activities at multiple levels. Although much has been learned about the biochemical reactions mediating cleavage and polyadenylation of pre-mRNAs, how alternative $3^{\prime}$ ends of pre-mRNAs are selected in vivo remains poorly understood. Here, guided by the genetic interactions

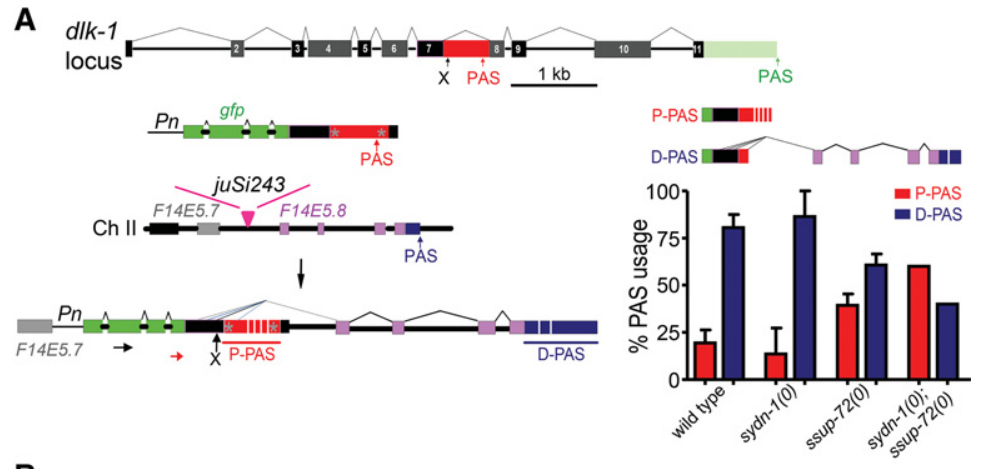

B

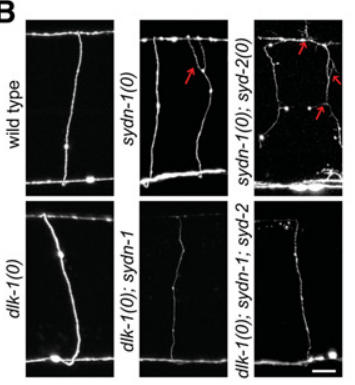

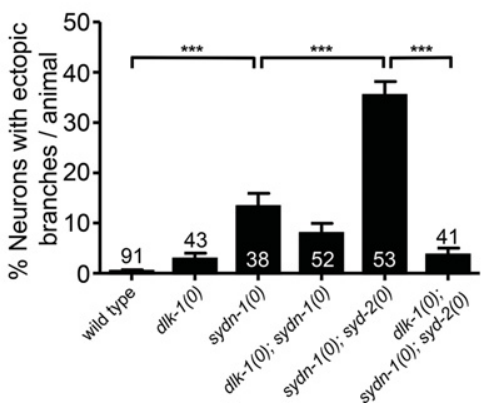

Figure 7. Dysregulated APA of dlk-1 contributes to neuronal defects of sydn-1 mutants. (A) Loss of function in ssup-72 can promote the usage of the internal dlk-1S PAS. (Left panel) Illustration of the GFP reporter for the dlk-1S PAS (juSi243) inserted on chromosome II. The F14E5.8 gene follows the insertion site. Splice sites of the PAS-containing intron are mutated (gray asterisks). Cryptic splice sites and PASs are labeled. Primers for 3'RACE are shown. (Right panel) Quantification of sequencing results from $3^{\prime}$ RACE products of the juSi243 reporter in the genotypes indicated. 3 RACE products were cloned into pCR 8 . For each genotype, $>20$ colonies were sequenced (for wild type, $n$ $=22$; for $\operatorname{sydn}-1(0), n=22$; for $\operatorname{ssup}-72(0), n=23$; for ssup-72(0); sydn-1(0), $n=20)$. (X) Stop codon. (B) Ectopic branches of GABAergic motor neurons in sydn-1(0) are suppressed by $d l k-1(0)$. Shown at the left are images of axon morphology of the GABAergic motor neurons labeled by juIs76 [Punc-25-GFP] in the genotypes indicated. Red arrows indicate abnormal branches. The graph at the right shows the quantification of the average percentage of GABAergic neurons that display ectopic branches in the genotypes indicated. Five to six commissures per L1 animal were counted. $\left({ }^{* * *}\right) P<$ 0.001, Kruskal-Wallis test and Dunn's multiple comparison post-test. Bar, $10 \mu \mathrm{m}$. 
between sydn-1 and ssup-72 or select NpolyA factors, we uncovered a molecular mechanism regulating coding region APA of two genes important for the development of the nervous system. We propose that the interaction between SYDN-1 and SSUP-72 at internal PASs regulates APA in a context-dependent manner (Supplemental Fig. S11).

\section{SSUP-72 controls APA within coding regions}

Ankyrins provide scaffolds for cells and act as adaptors for signaling pathways (Cunha and Mohler 2009). In C. elegans, the unc-44 locus generates multiple isoforms of ankyrin, using APA to meet the needs of different cell types (Otsuka et al. 2002). Transcripts encoding the neuron-specific UNC-44F isoform are reduced in sydn-1(0) mutants, and this reduction is restored to wild-type levels by loss of function in ssup-72 or select NpolyA factors (Fig. 4; data not shown). Using PAS reporters that create a competitive APA configuration between the coding region PAS of unc-44 and the PAS of unc-54 at the distal location, we show that unc-44 coding region PAS1 and PAS2 are strong PASs for $3^{\prime}$ end processing. In ssup-72(0) mutants, the use of the coding region unc-44 PAS2 is weakened, enabling transcription to proceed and terminate at a distal PAS regardless of the distance or sequence nature of the distal PAS. In $s y d n-1(0)$ mutants, transcription terminates at PAS2 of the endogenous unc-44 locus, and the production of the unc-44f mRNA is reduced. The observed function of SSUP-72 is highly specific to PAS2, as the usage of unc-44 PAS1, which also is a strong PAS for $3^{\prime}$ end processing, is unaffected by ssup-72(0). Furthermore, compared with wild type, the ratio of unc- $44 f$ mRNA to unc-44c mRNA is lower in sydn-1(0) and higher in ssup72(0) or ssup-72(0); sydn-1(0), suggesting that SYDN-1 may function to balance SSUP-72 activity at the coding region PAS2 of unc-44 to promote $3^{\prime}$ end processing of distal PASs. Importantly, we show that polyadenylation of unc$44 \mathrm{f}$ mRA is restored in genetic mutants in which usage of PAS2 is compromised, resulting in behavioral and neuronal morphology suppression of $s y d n-1(0)$ animals.

The negative regulation of SSUP-72 by SYDN-1 can also promote the expression of the $d l k-1 S$ isoform. The $d l k-1 S$ PAS contains a noncanonical polyadenylation hexamer. In our reporter assay, the $d l k-1 S$ PAS behaves as a weak signal for $3^{\prime}$ end processing, and loss of function in ssup72 facilitates the use of the $d l k-1 S$ PAS. The usage of the weak $d l k-1 S$ PAS appears to depend on the splicing of the intron containing the $d l k-1 S$ PAS, suggesting that splicing and 3 ' end processing are coordinated at this PAS. Our genetic studies demonstrate that dysregulation of APA in dlk-1 results in functional defects, consistent with its roles in neuronal development. Future studies will tease apart the molecular underpinning for such context-dependent regulation of APA.

\section{Regulation of RNA Pol II CTD phosphorylation is coupled with coding region APA}

The phosphorylation and dephosphorylation cycle on RNA Pol II CTD has been proposed to provide codes for transcription-coupled pre-mRNA processing (Egloff and Murphy 2008; Buratowski 2009). The interplay between CTD kinases and phosphatases therefore strongly affects the status of mRNA transcription at multiple steps, as differentially phosphorylated CTD can recruit interacting proteins and affect the dynamics of transcription-coupled events. For example, Thr4 phosphorylation of the CTD by CDK9 is required for histone mRNA 3 ' end processing (Hsin et al. 2011). Ser2 phosphorylation of the CTD is required for its association with polyadenylation factors (such as Pcf11) and for $3^{\prime}$ end processing of pre-mRNAs in yeast (Licatalosi et al. 2002; Ahn et al. 2004; Meinhart and Cramer 2004). The ratio of Ser5 phosphorylated CTD to Ser2 phosphorylated CTD is high at $5^{\prime}$ ends and low at $3^{\prime}$ ends (Komarnitsky et al. 2000). Ser7 phosphorylation of the CTD coincides with introns in budding yeast (Kim et al. 2010a). Ssu72 has a high degree of specificity for CTD Ser5 (Krishnamurthy et al. 2004; Xiang et al. 2010), although low activity on Ser7 has also been observed (Bataille et al. 2012; Xiang et al. 2012; Zhang et al. 2012). In human embryonic stem cells, Ssu72 is detected at introns by ChIP-seq (Chen et al. 2014). We speculate that dephosphorylation on Ser5P or Ser7P of the CTD at internal PASs by SSUP-72 helps to recruit select NpolyA factors and regulate $3^{\prime}$ end processing at internal PASs.

In other organisms, the phosphatase activity of Ssu72 is regulated by its binding partners. In yeast, Ssu72 binds Ptal (He et al. 2003), whereas, in mammals, Ssu72 binds symplekin. Symplekin and Ptal share weak sequence similarity, but both can facilitate Ssu72 phosphatase activity (Krishnamurthy et al. 2004; Xiang et al. 2010). We found that loss of function in symk-1 also suppresses sydn-1(0), consistent with C. elegans SYMK-1 being a functional partner of SSUP-72. Our protein interaction studies show that the presence of SYDN-1 reduces Pol II-associated Ssu72. Therefore, SYDN-1 may regulate SSUP-72 phosphatase activity by interfering with its interaction with Pol II.

In yeast, Ssu72 is essential for viability (Sun and Hampsey 1996), presumably because of its multiple functions in transcription (Dichtl et al. 2002; Steinmetz and Brow 2003; Krishnamurthy et al. 2004; Reyes-Reyes and Hampsey 2007). In contrast, we found that C. elegans SSUP-72 is dispensable for viability, fertility, and general development of the animals and instead plays a highly specific regulatory role in coding region APA. The growth defects of yeast ssu72 cells are suppressed by rpb2 and 6-azauracil, a drug impairing the enzyme required for synthesis of UTP and GTP, leading to the hypothesis that Ssu72 may normally attenuate the elongation rate of RNA Pol II (Dichtl et al. 2002). Although we did not observe an overall alteration of the CTD-binding pattern in ssup-72(0) mutants, high-resolution analysis of RNA Pol II CTD occupancy at the unc-44 PAS2 cassette revealed a noticeable peak shift in ssup-72(0) mutants (Fig. 5A). It is possible that SSUP-72 affects transcription elongation rates at internal PASs. Additionally, chromatin microenvironments at internal PASs of unc-44 or dlk-1 may contribute to increased pausing of RNA Pol II or successful splicing to allow differential regulation of internal PASs. 


\section{Regulatory role of NpolyA factors in neuronal APA}

The other NpolyA factors identified as genetic suppressors of sydn-1(0) either are in the CPSF complex, which functions in PAS recognition, or are CTD-binding proteins (Van Epps et al. 2010; and this study). While null mutants for these NpolyA genes are lethal and sterile, the development of the nervous system is remarkably normal. Conceivably, maternal contribution may provide sufficient activity for these proteins in neuronal differentiation and maintenance; we favor an alternative interpretation that select core NpolyA factors have substantial redundancy, and therefore elimination of individual factors is not detrimental for pre-mRNA processing in neurons. Null mutations in these NpolyA genes suppress sydn-1(0) to the same extent as ssup-72(0). Moreover, overexpression of an individual factor cannot bypass the requirement of the other. Thus, these proteins also function in a mutually dependent manner to coordinate APA, acting to terminate the transcription at the strong internal unc-44 PAS2 or promoting splicing at the weak internal $d l k-1 S$ PAS. Consistent with this idea, recent studies of mammalian CPSFs have linked their function to RNA Pol II pausing (Nag et al. 2007) and splicing (Martinson 2011; Misra et al. 2015).

Studies in human cells have identified $>85$ proteins as components of the $3^{\prime}$ processing complex, among which 50 additional proteins may mediate coupling with other cellular processes (Shi et al. 2009). Genetically, SYDN-1 interacts with NpolyA machinery but is specifically required for neuronal development. Importantly, from global assessment of polyadenylation events and genome-wide CTD occupancy assays, we did not detect major defects in PAS recognition in $s y d n-1(0)$, indicating that SYDN-1 does not alter the basal function of transcription and 3 ' end processing. Instead, SYDN-1 controls APA in neurons by modifying the phosphorylation state of the RNA Pol II CTD at intronic PASs. Overall, our findings reveal a previously unknown mechanism controlling coding region APA for transcript diversification in the nervous system.

\section{Materials and methods}

\section{C. elegans genetics}

Strains were maintained on NGM plates at $20^{\circ} \mathrm{C}-22.5^{\circ} \mathrm{C}$ as described (Brenner 1974). Genetic mutations used in this study are summarized in Supplemental Table S1. Double and triple mutants were constructed following standard procedures, and the genotypes were confirmed by DNA sequencing or restriction enzyme digestion of PCR fragments amplified using primers listed in Supplemental Table S1. Supplemental Table S2 lists all of the transgenes and strains used.

Transgenes of extrachromosomal arrays were generated following standard procedures (Mello et al. 1991). Expression constructs were injected at $1-20 \mathrm{ng} / \mathrm{\mu L}$ with coinjection marker Pttx-3-RFP at $50 \mathrm{ng} / \mathrm{\mu L}$ and pBlueScript to bring the total plasmid concentration to $100 \mathrm{ng} / \mathrm{\mu L}$. For each construct, two to three independent transgenic lines were analyzed (Supplemental Table S2). MosSCI lines were generated at the chromosome II ttTi5605 site using EG6699 as described (Frokjaer-Jensen et al. 2008). Single-copy insertions were verified by LongAmp amplification using primers designed by $\mathrm{M}$. Nonet (http://thalamus.wustl.edu/nonetlab/ resourcesf/resources.html).

\section{Fluorescence microscopy for synapse and axon morphology}

Synaptic puncta were scored under the Zeiss Axioskop from the entire dorsal cord of strains containing juIs1 [Punc-25-SNB-1:: GFP]. Axonal branches of GABAergic motor neurons visualized by juIs76 [Punc-25-GFP] were counted from five to six commissures of L1 worms or 15-19 commissures of adult worms. Percentages of neurons with ectopic branches per animal were counted by dividing commissure number with abnormal branches by total commissure number scored from each worm. For quantitative confocal imaging, 1-d-old adult worms were paralyzed in M9 buffer containing 0.5\%-1\% 1-phenoxy-2-propanol (TCL America). A Zeiss LSM510 or LSM710 confocal microscope equipped with Chroma HQ filters was used for collecting images. To compare the intensity of immunostaining or GFP reporter genes from different strains, we imaged the dorsal or ventral nerve cord around the vulval region. Images were processed using MetaMorph software with genotypes blinded to the observer. Average fluorescence intensity in axons was measured after drawing a line along the dorsal nerve cord and subtracting the background from the nearby area; average nuclear fluorescence intensity was measured after outlining individual nuclei and subtracting the background. The average fluorescence intensity of mutants was normalized to that of wild type.

\section{RT-PCR and 3'RACE analyses of $m R N A$}

Total RNA was isolated using TRIzol (Invitrogen) from synchronized L1 or L2 worms cultured under the same conditions. Total RNA $(2-5 \mu \mathrm{g})$ was reverse-transcribed into $20 \mu \mathrm{L}$ of cDNA library using an oligo dT primer (Invitrogen, 18080-051) or an adaptor primer containing oligo dT sequences (Invitrogen, 18373-019). For semiquantitative PCR amplification, 1/20 vol of cDNA library, amplified using oligo dT primer, was used. RT-qPCR was conducted using the iQ SYBR Green Supermix kit (Bio-Rad) on a CFX96TM real-time system (C1000TM ThermalCycler, BioRad). For each RT-qPCR reaction, 1/60 vol of cDNA library, amplified using oligo dT primer, was mixed with iQTM SYBR Green Supermix. Triplicate reactions were performed for each primer pair. The internal control rps-25 was done in parallel with other primer pairs. Data were analyzed using CFX manager software (version 2, Bio-Rad). Primers for detecting transcripts of ssup72, ama-1, unc-44, gfp, and rps-25 in RT-PCR or RT-qPCR are listed in Supplemental Table S3. In 3'RACE, gene-specific or nested primers listed in Supplemental Table S3 were used together with adaptor primers for the first or second round of PCR amplifications of 3 ' ends of unc-44, dlk-1, and $g f p$ reporters. In the first amplification, $1 / 20 \mathrm{vol}$ of cDNA library, amplified using adaptor primer, was used, and $2 \mu \mathrm{L}$ of the first PCR product was used in the second amplification.

\section{Statistical analysis}

Statistical significance between two samples was determined in Prism (GraphPad Software) by the two-tailed Student's $t$-test if the samples followed Gaussian distribution or the Mann-Whitney test if they did not. To compare multiple samples, the statistical significance between any two samples was determined by one-way ANOVA if all samples followed Gaussian distribution; otherwise, Kruskal-Wallis test was used. To determine how two specific samples differed in multiple samples, a post-hoc test of Bonferroni's or Dunn's multiple comparison was conducted after 
one-way ANOVA or Kruskal-Wallis test. For quantification of Western blot and RT-qPCR, paired $t$-test or one-way ANOVA was used.

\section{3' UTRome profiling and Pol II occupancy assay}

3' UTR libraries were constructed and sequenced as described (Mangone et al. 2010). ChIP-seq was performed as described (Mukhopadhyay et al. 2008). Additional details and data analyses are in the Supplemental Material.

\section{Cell culture, transfection, and coimmunoprecipitation}

HEK293T cells were cultured in Dulbecco's modified Eagle's medium (DMEM). Full-length sydn-1 and human ssu 72 cDNAs were cloned into pcDNA3-Flag to generate tagged proteins. Cells were transfected by X-tremeGENE 9 DNA transfection reagent (Roche). Three days after transfection, cell lysates were prepared in RIPA buffer (25 mM Tris- $\mathrm{HCl}$ at pH 7.4, $150 \mathrm{mM} \mathrm{KCl}, 5 \mathrm{mM}$ EDTA, $1 \%$ NP- $40,1 \%$ sodium deoxycholate, $0.1 \%$ SDS) with protease inhibitor cocktail (Roche) and phosphatase inhibitor cocktails (Sigma, P0044 and P5726). Equivalent amounts of lysates were incubated with anti-Pol II-bound (N-20, Santa Cruz Biotechnology, sc-899X) Dynabeads Protein A/G (Novex by Life Technologies) overnight at $4^{\circ} \mathrm{C}$. After four washes, the bound proteins were eluted by heating for $5 \mathrm{~min}$ at $95^{\circ} \mathrm{C}$ in $2 \times$ Laemmli sample buffer (Bio-Rad) and resolved by SDS-PAGE. The coimmunoprecipitated proteins were blotted with primary mouse anti-Flag (Sigma, F3165), rabbit anti-Pol II (N-20; Santa Cruz Biotechnology, sc899X), or mouse anti-Pol II (CTD4H8; Santa Cruz Biotechnology, sc-47701) antibody and visualized with HRP-conjugated antimouse or anti-rabbit secondary antibody (Amersham) at 1:5000 dilution using SuperSignal West Pico kit (Thermo Scientific).

\section{Yeast two-hybrid assay}

Bait and prey were cloned into pACT2 containing the GAL4 activation domain and pBTM166 containing the GAL4 DNAbinding domain (Clontech). Plasmids encoding activation domain fused SSUP-72 (pCZGY2665) or activation domain-only control were cotransformed with plasmids encoding the binding domain fused full-length SYDN-1 (pCZGY2666), the N-terminal half of SYDN-1 (pCZGY2667), or the C-terminal half of SYDN-1 (pCZGY2668) or the binding domain only into the L40 yeast strain and selected on Trp ${ }^{-} \mathrm{Leu}^{-}$plates to obtain cotransformants. Single colonies were picked from each plate and cultured until $\mathrm{OD}_{600}=0.4-1.0$. Yeast cells were pelleted by centrifugation, washed twice with $\mathrm{ddH}_{2} \mathrm{O}$, and resuspended in $\mathrm{ddH}_{2} \mathrm{O}$. Five microliters of yeast cells per spot was plated on Histidine selection plates with or without $1 \mathrm{mM} 3 \mathrm{AT}$ in a dilution series.

Generation of unc-44(ju1377) and unc-44(ju1378)

by CRISPR/Cas9-mediated genome editing

We designed two sgRNAs around the polyadenylation consensus sequence (marked red in Supplemental Table S4) in the PAS2 cassette of unc-44 (ATTAACCGACATATACGAAT and GTTCCA ATTGCTCCTGCCAT). We generated sgRNA plasmids using the Peft-3-cas9-NLS-pU6-sgRNA vector (pDD162; Addgene, \#47549) by PCR-based site-directed mutagenesis as described (Dickinson et al. 2013). A mixture of Peft-3-cas9-NLS-pU6sgRNA plasmids (100 ng/ $\mathrm{LL}$ each pCZGY2855 and pCZGY2856) and $5 \mathrm{ng} / \mu \mathrm{L}$ Pmyo-3-mCherry (as a coinjection marker) was injected into 30 P0 1-d-old adults of sydn-1(0); syd-2(0); juIs76. We obtained $16 \mathrm{~F} 1 \mathrm{~s}$ with mCherry expression. From the progeny of two F1s, we observed worms showing improved locomotion and isolated them as ju1377 and ju1378. We determined by DNA sequence analysis that ju1377 and ju1378 contained 83bp and 180-bp deletions in the PAS2 cassette, respectively (highlighted in Supplemental Table S4).

\section{Acknowledgments}

We thank Rui Xiao for providing primers and protocols of RNA Pol II CTD ChIP-seq, Anthony Otsuka and Lihsia Chen for providing antibodies for UNC-44, Ya Dai for her contribution to the isolation of several sydn-1(0) suppressors, Dong Yan for discussion on $d l k-1 S$, and Shohei Mitani (the Japanese National Bioresource Project) and the C. elegans gene knockout consortium for deletion alleles. We thank members of the Jin and Chisholm laboratories for help and comments. Some strains were obtained from the Caenorhabditis Genetics Center, which is supported by National Institutes of Health P40 OD010440. We acknowledge WormBase for information resources. This work was supported by National Institutes of Health grants R01 NS035546 to Y.J., R01GM088565 to J.K., and R01 GM049369 to X.F. F.C. is an associate and Y. J. is an investigator of the Howard Hughes Medical Institute.

\section{References}

Ahn SH, Kim M, Buratowski S. 2004. Phosphorylation of serine 2 within the RNA polymerase II C-terminal domain couples transcription and $3^{\prime}$ end processing. Mol Cell 13: 67-76.

Bataille AR, Jeronimo C, Jacques PE, Laramee L, Fortin ME, Forest A, Bergeron M, Hanes SD, Robert F. 2012. A universal RNA polymerase II CTD cycle is orchestrated by complex interplays between kinase, phosphatase, and isomerase enzymes along genes. Mol Cell 45: 158-170.

Berg MG, Singh LN, Younis I, Liu Q, Pinto AM, Kaida D, Zhang Z, Cho S, Sherrill-Mix S, Wan L, et al. 2012. U1 snRNP determines mRNA length and regulates isoform expression. Cell 150: 53-64.

Boontrakulpoontawee P, Otsuka AJ. 2002. Mutational analysis of the Caenorhabditis elegans ankyrin gene unc-44 demonstrates that the large spliceoform is critical for neural development. Mol Genet Genomics 267: 291-302.

Brenner S. 1974. The genetics of Caenorhabditis elegans. Genetics 77: 71-94.

Buratowski S. 2009. Progression through the RNA polymerase II CTD cycle. Mol Cell 36: 541-546.

Chan SL, Huppertz I, Yao C, Weng L, Moresco JJ, Yates JR III, Ule J, Manley JL, Shi Y. 2014. CPSF30 and Wdr33 directly bind to AAUAAA in mammalian mRNA $3^{\prime}$ processing. Genes Dev 28: 2370-2380.

Chen Y, Zhang L, Estaras C, Choi SH, Moreno L Jr, Karn J, Moresco JJ, Yates JR III, Jones KA. 2014. A gene-specific role for the Ssu72 RNAPII CTD phosphatase in HIV-1 Tat transactivation. Genes Dev 28: 2261-2275.

Cheng C, Sharp PA. 2003. RNA polymerase II accumulation in the promoter-proximal region of the dihydrofolate reductase and $\gamma$-actin genes. Mol Cell Biol 23: 1961-1967.

Colgan DF, Manley JL. 1997. Mechanism and regulation of mRNA polyadenylation. Genes Dev 11: 2755-2766.

Cui M, Allen MA, Larsen A, Macmorris M, Han M, Blumenthal T. 2008. Genes involved in pre-mRNA $3^{\prime}$-end formation and transcription termination revealed by a lin-15 operon Muv suppressor screen. Proc Natl Acad Sci 105: 16665-16670. 
Cunha SR, Mohler PJ. 2009. Ankyrin protein networks in membrane formation and stabilization. I Cell Mol Med 13: 4364-4376.

Davidson L, Muniz L, West S. 2014. 3' end formation of premRNA and phosphorylation of Ser2 on the RNA polymerase II CTD are reciprocally coupled in human cells. Genes Dev 28: 342-356.

Dichtl B, Blank D, Ohnacker M, Friedlein A, Roeder D, Langen H, Keller W. 2002. A role for SSU72 in balancing RNA polymerase II transcription elongation and termination. Mol Cell 10: 1139-1150.

Dickinson DJ, Ward JD, Reiner DJ, Goldstein B. 2013. Engineering the Caenorhabditis elegans genome using Cas9-triggered homologous recombination. Nat Methods 10: 1028-1034.

Di Giammartino DC, Nishida K, Manley JL. 2011. Mechanisms and consequences of alternative polyadenylation. Mol Cell 43: $853-866$.

Egloff S, Murphy S. 2008. Cracking the RNA polymerase II CTD code. Trends Genet 24: 280-288.

Elkon R, Ugalde AP, Agami R. 2013. Alternative cleavage and polyadenylation: extent, regulation and function. Nat Rev Genet 14: 496-506.

Flavell SW, Kim TK, Gray JM, Harmin DA, Hemberg M, Hong EJ, Markenscoff-Papadimitriou E, Bear DM, Greenberg ME. 2008. Genome-wide analysis of MEF2 transcriptional program reveals synaptic target genes and neuronal activity-dependent polyadenylation site selection. Neuron 60: 1022-1038.

Frokjaer-Jensen C, Davis MW, Hopkins CE, Newman BJ, Thummel JM, Olesen SP, Grunnet M, Jorgensen EM. 2008. Singlecopy insertion of transgenes in Caenorhabditis elegans. Nat Genet 40: 1375-1383.

He X, Khan AU, Cheng H, Pappas DL Jr, Hampsey M, Moore CL. 2003. Functional interactions between the transcription and mRNA $3^{\prime}$ end processing machineries mediated by $\mathrm{Ssu} 72$ and Sub1. Genes Dev 17: 1030-1042.

Hilgers V, Lemke SB, Levine M. 2012. ELAV mediates 3' UTR extension in the Drosophila nervous system. Genes Dev 26: 2259-2264.

Hirose Y, Manley JL. 1998. RNA polymerase II is an essential mRNA polyadenylation factor. Nature 395: 93-96.

Hsin JP, Sheth A, Manley JL. 2011. RNAP II CTD phosphorylated on threonine-4 is required for histone mRNA $3^{\prime}$ end processing. Science 334: 683-686.

Jenal M, Elkon R, Loayza-Puch F, van Haaften G, Kuhn U, Menzies FM, Oude Vrielink JA, Bos AJ, Drost J, Rooijers K, et al. 2012. The poly(A)-binding protein nuclear 1 suppresses alternative cleavage and polyadenylation sites. Cell 149: 538-553.

Kaida D, Berg MG, Younis I, Kasim M, Singh LN, Wan L, Dreyfuss G. 2010. U1 snRNP protects pre-mRNAs from premature cleavage and polyadenylation. Nature 468: 664-668.

Kim M, Krogan NJ, Vasiljeva L, Rando OJ, Nedea E, Greenblatt JF, Buratowski S. 2004. The yeast Rat1 exonuclease promotes transcription termination by RNA polymerase II. Nature 432: 517-522.

Kim H, Erickson B, Luo W, Seward D, Graber JH, Pollock DD, Megee PC, Bentley DL. 2010a. Gene-specific RNA polymerase II phosphorylation and the CTD code. Nat Struct Mol Biol 17: 1279-1286.

Kim S, Yamamoto J, Chen Y, Aida M, Wada T, Handa H, Yamaguchi Y. 2010b. Evidence that cleavage factor Im is a heterotetrameric protein complex controlling alternative polyadenylation. Genes Cells 15: 1003-1013.

Kolev NG, Yario TA, Benson E, Steitz JA. 2008. Conserved motifs in both CPSF73 and CPSF100 are required to assemble the ac- tive endonuclease for histone mRNA $3^{\prime}$-end maturation. EMBO Rep 9: 1013-1018.

Komarnitsky P, Cho EJ, Buratowski S. 2000. Different phosphorylated forms of RNA polymerase II and associated mRNA processing factors during transcription. Genes Dev 14: 24522460.

Krishnamurthy S, He X, Reyes-Reyes M, Moore C, Hampsey M. 2004. Ssu72 Is an RNA polymerase II CTD phosphatase. Mol Cell 14: 387-394.

Lianoglou S, Garg V, Yang JL, Leslie CS, Mayr C. 2013. Ubiquitously transcribed genes use alternative polyadenylation to achieve tissue-specific expression. Genes Dev 27: 2380-2396.

Licatalosi DD, Geiger G, Minet M, Schroeder S, Cilli K, McNeil JB, Bentley DL. 2002. Functional interaction of yeast premRNA $3^{\prime}$ end processing factors with RNA polymerase II. Mol Cell 9: 1101-1111.

Licatalosi DD, Mele A, Fak JJ, Ule J, Kayikci M, Chi SW, Clark TA, Schweitzer AC, Blume JE, Wang X, et al. 2008. HITSCLIP yields genome-wide insights into brain alternative RNA processing. Nature 456: 464-469.

Mangone M, Manoharan AP, Thierry-Mieg D, Thierry-Mieg J, Han T, Mackowiak SD, Mis E, Zegar C, Gutwein MR, Khivansara V, et al. 2010. The landscape of C. elegans 3'UTRs. Science 329: 432-435.

Martin G, Gruber AR, Keller W, Zavolan M. 2012. Genome-wide analysis of pre-mRNA $3^{\prime}$ end processing reveals a decisive role of human cleavage factor I in the regulation of $3^{\prime}$ UTR length. Cell Rep 1: 753-763.

Martinson HG. 2011. An active role for splicing in 3'-end formation. Wiley Interdiscip Rev RNA 2: 459-470.

Masamha CP, Xia Z, Yang J, Albrecht TR, Li M, Shyu AB, Li W, Wagner EJ. 2014. CFIm25 links alternative polyadenylation to glioblastoma tumour suppression. Nature 510: 412-416.

Mayr C, Bartel DP. 2009. Widespread shortening of 3'UTRs by alternative cleavage and polyadenylation activates oncogenes in cancer cells. Cell 138: 673-684.

McCracken S, Fong N, Yankulov K, Ballantyne S, Pan G, Greenblatt J, Patterson SD, Wickens M, Bentley DL. 1997. The C-terminal domain of RNA polymerase II couples mRNA processing to transcription. Nature 385: 357-361.

Meinhart A, Cramer P. 2004. Recognition of RNA polymerase II carboxy-terminal domain by $3^{\prime}$-RNA-processing factors. $\mathrm{Na}$ ture 430: 223-226.

Meinhart A, Kamenski T, Hoeppner S, Baumli S, Cramer P. 2005. A structural perspective of CTD function. Genes Dev 19: 1401-1415.

Mello CC, Kramer JM, Stinchcomb D, Ambros V. 1991. Efficient gene transfer in C.elegans: extrachromosomal maintenance and integration of transforming sequences. EMBO $J$ 10: 3959-3970.

Millevoi S, Vagner S. 2010. Molecular mechanisms of eukaryotic pre-mRNA $3^{\prime}$ end processing regulation. Nucleic Acids Res 38: $2757-2774$.

Misra A, Ou J, Zhu LJ, Green MR. 2015. Global promotion of alternative internal exon usage by mRNA $3^{\prime}$ end formation factors. Mol Cell 58: 819-831.

Mukhopadhyay A, Deplancke B, Walhout AJ, Tissenbaum HA. 2008. Chromatin immunoprecipitation (ChIP) coupled to detection by quantitative real-time PCR to study transcription factor binding to DNA in Caenorhabditis elegans. Nat Protoc 3: 698-709.

Nag A, Narsinh K, Martinson HG. 2007. The poly(A)-dependent transcriptional pause is mediated by CPSF acting on the body of the polymerase. Nat Struct Mol Biol 14: 662-669. 
Nakata K, Abrams B, Grill B, Goncharov A, Huang X, Chisholm AD, Jin Y. 2005. Regulation of a DLK-1 and p38 MAP kinase pathway by the ubiquitin ligase RPM-1 is required for presynaptic development. Cell 120: 407-420.

Otsuka AJ, Boontrakulpoontawee P, Rebeiz N, Domanus M, Otsuka D, Velamparampil N, Chan S, Vande Wyngaerde M, Campagna S, Cox A. 2002. Novel UNC-44 AO13 ankyrin is required for axonal guidance in C. elegans, contains six highly repetitive STEP blocks separated by seven potential transmembrane domains, and is localized to neuronal processes and the periphery of neural cell bodies. I Neurobiol 50: 333349.

Phatnani HP, Greenleaf AL. 2006. Phosphorylation and functions of the RNA polymerase II CTD. Genes Dev 20: 2922-2936.

Proudfoot NJ. 2011. Ending the message: poly(A) signals then and now. Genes Dev 25: 1770-1782.

Reyes-Reyes M, Hampsey M. 2007. Role for the Ssu72 C-terminal domain phosphatase in RNA polymerase II transcription elongation. Mol Cell Biol 27: 926-936.

Sandberg R, Neilson JR, Sarma A, Sharp PA, Burge CB. 2008. Proliferating cells express mRNAs with shortened 3 ' untranslated regions and fewer microRNA target sites. Science 320: 1643-1647.

Schonemann L, Kuhn U, Martin G, Schafer P, Gruber AR, Keller W, Zavolan M, Wahle E. 2014. Reconstitution of CPSF active in polyadenylation: recognition of the polyadenylation signal by WDR33. Genes Dev 28: 2381-2393.

Shi Y, Di Giammartino DC, Taylor D, Sarkeshik A, Rice WJ, Yates JR III, Frank J, Manley JL. 2009. Molecular architecture of the human pre-mRNA 3' processing complex. Mol Cell 33: 365-376.

Steinmetz EJ, Brow DA. 2003. Ssu72 protein mediates both poly (A)-coupled and poly(A)-independent termination of RNA polymerase II transcription. Mol Cell Biol 23: 6339-6349.

St-Pierre B, Liu X, Kha LC, Zhu X, Ryan O, Jiang Z, Zacksenhaus E. 2005. Conserved and specific functions of mammalian ssu72. Nucleic Acids Res 33: 464-477.

Sullivan KD, Steiniger M, Marzluff WF. 2009. A core complex of CPSF73, CPSF100, and Symplekin may form two different cleavage factors for processing of poly(A) and histone mRNAs. Mol Cell 34: 322-332.

Sun ZW, Hampsey M. 1996. Synthetic enhancement of a TFIIB defect by a mutation in SSU72, an essential yeast gene encoding a novel protein that affects transcription start site selection in vivo. Mol Cell Biol 16: 1557-1566.

Takagaki Y, Seipelt RL, Peterson ML, Manley JL. 1996. The polyadenylation factor CstF-64 regulates alternative processing of IgM heavy chain pre-mRNA during B cell differentiation. Cell 87: 941-952.

Tian B, Hu J, Zhang H, Lutz CS. 2005. A large-scale analysis of mRNA polyadenylation of human and mouse genes. Nucleic Acids Res 33: 201-212.

Van Epps H, Dai Y, Qi Y, Goncharov A, Jin Y. 2010. Nuclear premRNA $3^{\prime}$-end processing regulates synapse and axon development in C. elegans. Development 137: 2237-2250.

Vasiljeva L, Kim M, Mutschler H, Buratowski S, Meinhart A. 2008. The Nrd1-Nab3-Sen 1 termination complex interacts with the Ser5-phosphorylated RNA polymerase II C-terminal domain. Nat Struct Mol Biol 15: 795-804.

Wallenfang MR, Seydoux G. 2002. cdk-7 is required for mRNA transcription and cell cycle progression in Caenorhabditis elegans embryos. Proc Natl Acad Sci 99: 55275532.

Xiang K, Nagaike T, Xiang S, Kilic T, Beh MM, Manley JL, Tong L. 2010. Crystal structure of the human symplekinSsu72-CTD phosphopeptide complex. Nature 467: 729733.

Xiang K, Manley JL, Tong L. 2012. An unexpected binding mode for a Pol II CTD peptide phosphorylated at Ser7 in the active site of the CTD phosphatase Ssu72. Genes Dev 26: 2265-2270.

Yan D, Jin Y. 2012. Regulation of DLK-1 kinase activity by calcium-mediated dissociation from an inhibitory isoform. Neuron 76: 534-548.

Zhang DW, Mosley AL, Ramisetty SR, Rodriguez-Molina JB, Washburn MP, Ansari AZ. 2012. Ssu72 phosphatase-dependent erasure of phospho-Ser7 marks on the RNA polymerase II C-terminal domain is essential for viability and transcription termination. J Biol Chem 287: 8541-8551. 


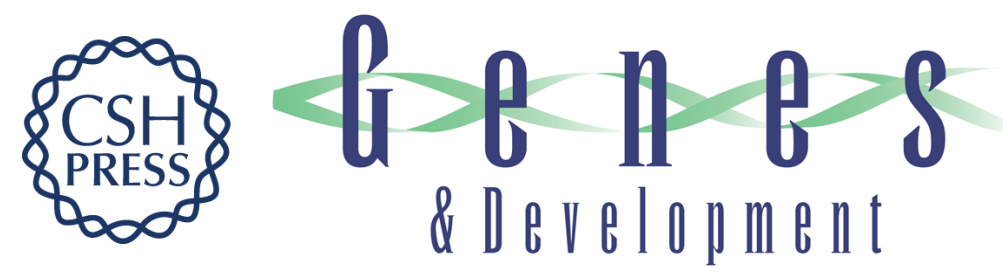

\title{
Context-dependent modulation of Pol II CTD phosphatase SSUP-72 regulates alternative polyadenylation in neuronal development
}

\author{
Fei Chen, Yu Zhou, Yingchuan B. Qi, et al.
}

Genes Dev. 2015, 29:

Access the most recent version at doi:10.1101/gad.266650.115

\section{Supplemental http://genesdev.cshlp.org/content/suppl/2015/11/19/29.22.2377.DC1 \\ Material}

References This article cites 69 articles, 26 of which can be accessed free at:

http://genesdev.cshlp.org/content/29/22/2377.full.html\#ref-list-1

Creative This article is distributed exclusively by Cold Spring Harbor Laboratory Press for the first

Commons six months after the full-issue publication date (see

License http://genesdev.cshlp.org/site/misc/terms.xhtml). After six months, it is available under a Creative Commons License (Attribution-NonCommercial 4.0 International), as described at http://creativecommons.org/licenses/by-nc/4.0/.

Email Alerting Receive free email alerts when new articles cite this article - sign up in the box at the top Service right corner of the article or click here.

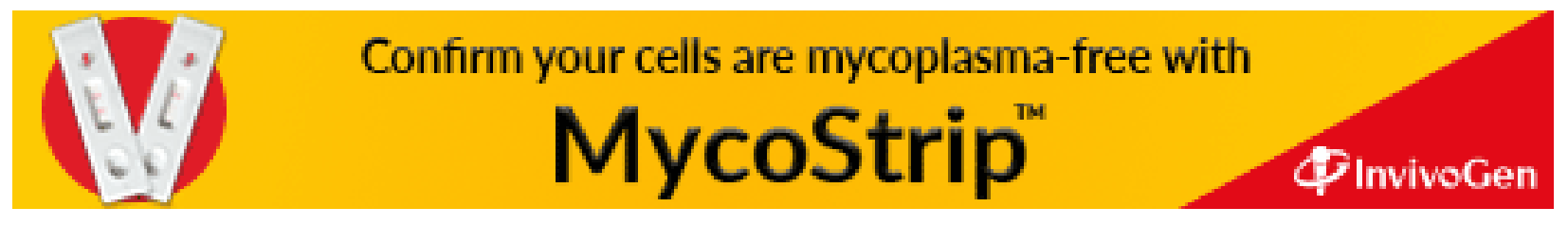

